\title{
The Changes in Static Balance During Pregnancy: A Prospective Longitudinal Study
}

\author{
Seyda Sancar ${ }^{1}(\mathbb{D})$, Nevin Atalay Guzel ${ }^{1}\left(\mathbb{D}\right.$, Gamze Cobanoglu ${ }^{1}(\mathbb{D})$, Yaprak Arzu Ozdemir ${ }^{2}\left(\mathbb{D}\right.$, Merih Bayram $^{3}(\mathbb{D})$ \\ ${ }^{1}$ Gazi University, Faculty of Health Sciences, Department of Physiotherapy and Rehabilitation, Ankara, Turkey. \\ ${ }^{2}$ Gazi University, Faculty of Science, Department of Statistics, Ankara, Turkey. \\ ${ }^{3}$ Gazi University, Faculty of Medicine, Department of Gynecology and Obstetrics, Ankara, Turkey. \\ Correspondence Author: Gamze Cobanoglu \\ E-mail: fztgamze7@gmail.com
}

Received: 28.05.2020 Accepted: 16.03.2021

\begin{abstract}
Objective: The aim of this study was to examine the changes in static balance during the three trimesters of pregnancy.

Methods: Nineteen pregnant women who were in the first trimester of pregnancy were included in the study. The static balance assessment of the pregnant women was performed with Biodex-BioSway ${ }^{\mathrm{TM}}$ Balance System. The balance was assessed with the Postural Stability Test, Limits of Stability Test (LOS) and Modified Clinical Test of Sensory Integration and Balance (mCTSIB). Measurements were repeated during the 1st trimester (10-12 weeks), the 2nd trimester (22-24 weeks) and the 3rd trimester (34th week) of the same pregnant women.

Results: There was no significant difference between trimesters in terms of the postural stability test $(p>0.05)$. It was determined that there was a difference between the three trimesters in the LOS scores of the pregnant women $(p<0.05)$. There was a significant increase in the LOS in the last trimester compared to the first trimester. According to the $\mathrm{mCTSIB}$, there was only a difference between the three trimesters in the eyes closed firm surface parameter $(p<0.05)$. Oscillations were higher in the third trimester than in the second trimester.

Conclusion: As a result of this study, which underlines the importance of evaluating static balance by follow-up of the same pregnant women during three trimesters, it is seen that there are changes in different parameters of balance. Considering the differences in the sub-parameters of balance in pregnant women; multi-directional evaluation involving different parameters may be beneficial when evaluating balances.

Keyword: pregnant, static balance, postural stability, limit of stability, modified sensory test
\end{abstract}

\section{INTRODUCTION}

During pregnancy, the body of the pregnant woman undergoes important anatomical and physiological changes in order to nurture and accommodate the developing fetus (1). These physiological changes may lead to alterations in the biomechanical structure of the pregnant woman resulting in changes in balance and postural control (2). The increase in body weight which occurs to meet the needs of the baby and to maintain the health of the mother, is one of the most important changes that occurs during pregnancy. An average increase of $11-16 \mathrm{~kg}$ is considered normal (3). Abdominal morphology changes from the second trimester, with the increasing size of the uterus and weight of the fetus, and there is a $30 \%$ increase in abdominal mass (4). The increase in abdomen size is related to decrease in static stability (5) and adaptive changes occurring in spinal curvatures which would compensate the anterior displacement of the center of gravity to maintain postural balance (6). It has been reported that gestational weight gain and the asymmetrical distribution of weight mainly in the anterior abdominal region (4), the adaptive postural changes necessary for readjustment of anterior-posterior center of gravity location (7), and increased joint laxity (8) may lead to changes in the static stability of a pregnant woman (5). Adaptations occurring in soft tissue, joints, and posture are important, both due to associated discomfort and pain, and due to changes in postural balance which increases the risk of falling (9). It is also stated that pregnant and postpartum women often suffer from sleep deprivation because they do not get enough sleep and this may also have an effect on postural stability (10). In pregnant women, it is very important to evaluate the changes in postural stability. Good postural control is essential for maintaining balance. In static posturography, increased sway of the human body during quiet standing may indicate a decrease in postural stability. An unstable posture may prevent optimal motor activity and cause falls (5).

It is stated that the above mentioned anatomical and physiological changes may cause postural instability during pregnancy. However, in the literature, there is no consensus 
among the studies evaluating pregnant women in terms of these parameters. Although there are studies evaluating pregnant women in terms of postural sway, to the best of our knowledge we believe that there is no study in literature evaluating all sub parameters of static balance in pregnant women during three trimesters. Based on the assumption that the static balance of pregnant woman who are followed up for three trimesters will decrease, the aim of this study is to examine the changes that occur in static balance during pregnancy.

\section{METHODS}

\subsection{Individuals}

This study was carried out in Gazi University Faculty of Health Sciences, Physiotherapy and Rehabilitation Department and Gazi University Faculty of Medicine Obstetrics and Gynecology Department. Approval was obtained from the Ethics Committee of Gazi University under the number 77082166-604.01.02-6834. Pregnant women were informed about the study in detail and written consent was obtained from each participant. Guidance and medical examinations of the pregnant women were carried out by an Obstetrics and Gynecology specialist at Gazi University.

Pregnant women between the ages of 18-40, who were in the first trimester of pregnancy (10-12) with a singleton live fetus, and without a pregnancy risk were included in the study. Exclusion criteria were; fetal developmental delay or anomalies, maternal problems such as; chronic diseases (hypertension, cancer, diabetes, vasculopathy), obesity in the first trimester (Body mass index (BMI)> 30), those who had neuromuscular, visual or orthopedic problems, and any problem which could affect balance were not included in the study. As a result, 19 pregnant women who met the criteria and agreed to participate in the study were included (Figure 1. Flow Diagram).

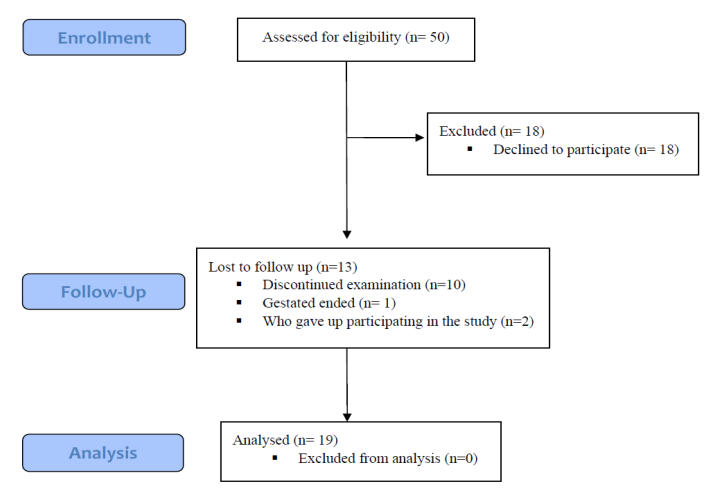

Figure 1. Flow diagram illustrating the participants in the study

The measurements were repeated 3 times in the same pregnant women; for 10-12 weeks (in the 1st trimester),
22-24 weeks (in the 2nd trimester) and 34th week-delivery (in the 3rd trimester).

Static balance was evaluated with Biodex-BioSway ${ }^{\mathrm{TM}}$ Balance System (BBS) (SD 950-340, Biodex Medical Systems, Inc., Shirley, NY, USA) (11). This device has 3 separate tests and 6 separate training programs and provides valid, reliable, and repeatable objectives measures of a patient's ability to balance on a firm and/or foam surface. Balance assessment with BBS was performed with the Postural Stability Test, Limits of Stability (LOS) and Modified clinical test of sensory integration and balance (mCTSIB).

The Postural Stability Test was used to evaluate the pregnant woman's ability to maintain center of balance. It was explained that the dot on the screen represents her Center of Gravity (CoG) and that she had to maintain her CoG by moving her body (without moving her feet) and hold it on the target point for 30 seconds. This test was repeated 3 times. As a result of the test, the Overall Stability Index (OSI), Medial-Lateral Stability Index (MLSI) and Anterior-Posterior Stability Index (APSI) were obtained. These scores indicate the amount of deviation from the anterior-posterior (AP) and medial-lateral (ML) axes. The patient's score on this test assesses deviations from center. Low scores indicate that the deviation is low, and the person's postural stability is good, thus a lower score is more desirable than a higher score.

LOS test is defined as the maximum angle a person's body can achieve from vertical without losing balance. The pregnant woman was asked to move the cursor representing her CoG to the targets on the screen ( 8 target points in the shape of a circle and 1 target point in the center). By shifting her weight, the pregnant woman was instructed to reach the blinking target and back as quickly and with as little deviation as possible. The test was repeated 3 times. As a result of the test, the "overall" score of the individual was obtained. A low "actual" score indicates that the person's LOS are decreased.

The mCTSIB test was used to evaluate the standing balance in different situations (eyes open / closed, firm support surface / dynamic support surface) that the individual may encounter in daily life. To reduce the effect of visual feedback on balance, the dot representing the center of gravity is not visible on the screen during the $\mathrm{mCTSIB}$. This test is performed in four different situations, with 30 seconds for each test and a 10 second rest period between each test. For the first situation, the pregnant woman was instructed to stand with her eyes open on the firm surface. For the second situation the test was performed on the same surface with the pregnant woman's eyes closed. For the third and fourth situations an foam surface was placed on the platform (to reduce the effect of the surface) and the test was performed with the pregnant woman's eyes open for the third situation and with her eyes closed for the fourth situation. As a result of the test, the body sway was calculated and the sway index scores (SI-Sway Index) for; Eyes Open Firm Surface, Eyes Closed Firm Surface, Eyes Open Foam Surface, Eyes Closed Foam Surface were obtained. A higher sway index score indicates that the person has increased postural sway. 


\subsection{Statistical analysis}

Statistical analyses of the study were carried out with SPSS for Windows 22.0 package program. The normal distribution of the data was analyzed with Tests of Normality and ShapiroWilk Test. The non-normal distributed data obtained from the measurements of pregnant women were presented using medians and interquartile range. In comparisons between trimesters, the statistical significance of the change over time for parameters was determined by Friedman test for the non-normal distributed data. Total type-1 error level was used as 0.05 for statistical significance. When necessary, the Bonferroni corrections for pairwise comparisons were evaluated with the Wilcoxon Test.

\section{RESULTS}

Based on a $96 \%$ power analysis, 19 pregnant women were included in this study. The average age of the pregnant women included was $29.31 \pm 5.57$ and the average height was $162.79 \pm 6.3 \mathrm{~cm}$. BMls of pregnant women are shown in Table 1. When BMI of pregnant women were evaluated, there was a significant difference between trimesters $(p<0.05$, Table 1). According to the Bonferroni correction, there was no significant difference between average pre-pregnancy BMI and 1st trimester BMI ( $p>0.008$, Table 1). However, all other binary comparisons were found to be statistically significant ( $p<0.008$, Table 1).
The changes in the static balances of pregnant women are given in Table 2. No statistically significant difference was found in the postural stability evaluations of pregnant women in terms of APSI, MLSI and OSI in all three trimesters ( $p>0.05$, Table 2). It was found that pregnant women showed similar postural stability values in all three trimesters.

There was a significant difference between the three trimesters in terms of the pregnant women's LOS scores; $(p<0.05$, Table 2$)$. According to the binary comparisons, it was seen that this difference was due to the 1st and 3rd trimesters. It was found that the LOS score was higher in the third trimester than in the first trimester. Likewise, although there was no statistically significant difference, the LOS score was found to be higher in the 3rd trimester than the 2nd trimester.

In the mCTSIB evaluation, it was found that in the comparison of Eyes Open Firm Surface, Eyes Closed Firm Surface, Eyes Open Foam Surface, Eyes Closed Foam Surface parameters between trimesters, there was only a difference in the Eyes Closed Firm Surface parameter and this difference was due to the difference between the $2 \mathrm{nd}$ and $3 \mathrm{rd}$ trimesters $(p<0.05$, Table 2). Differences between trimesters in other parameters were not statistically significant ( $p>0.05$, Table 2). It was observed that the amount of sway in Eyes Closed Firm Surface was greater in the third trimester compared to the second trimester.

Table 1. Body mass index changes of pregnant women

\begin{tabular}{|c|c|c|c|c|c|c|}
\hline$n=19$ & $\begin{array}{l}\text { Before Pregnancy } \\
\text { (0) }\end{array}$ & $\begin{array}{l}\text { 1.trimester } \\
\text { (1) }\end{array}$ & $\begin{array}{l}\text { 2.trimester } \\
\text { (2) }\end{array}$ & $\begin{array}{l}\text { 3.trimester } \\
\text { (3) }\end{array}$ & $p$ & Difference \\
\hline $\begin{array}{c}\text { BMI } \\
\left(\mathrm{kg} / \mathrm{m}^{2}\right)\end{array}$ & $\begin{array}{c}23,87 \\
(22,35 / 25,75)\end{array}$ & $\begin{array}{c}24,56 \\
(22,35 / 26,72)\end{array}$ & $\begin{array}{c}26,8 \\
(24,36 / 27,48)\end{array}$ & $\begin{array}{c}29,39 \\
(27,12 / 32,44)\end{array}$ & $0,000 *$ & $\begin{array}{l}0,012^{+} \text {for } 1-2 \\
0,000^{+} \text {for } 2-3 \\
0,000^{+} \text {for } 1-3 \\
0,000^{+} \text {for } 0-2 \\
0,000+\text { for } 0-3\end{array}$ \\
\hline
\end{tabular}

BMI: Body Mass Index, ${ }^{*} p<0.001,{ }^{+}$New $p$ value with Bonferroni correction $p / 6=0.05 / 6=0.008$

Table 2. The changes in static balance of pregnant women during three trimesters

\begin{tabular}{|c|c|c|c|c|c|}
\hline & 1.trimester & 2.trimester & 3.trimester & $p$ & Difference \\
\hline APSI & $0,2(0,1 / 0,2)$ & $0,2(0,1 / 0,2)$ & $0,2(0,2 / 0,2)$ & 0.203 & - \\
\hline MLSI & $0,1(0,1 / 0,2)$ & $0,1(0,1 / 0,1)$ & $0,1(0,1 / 0,1)$ & 0.807 & - \\
\hline OSI & $0,2(0,2 / 0,3)$ & $0,2(0,2 / 0,3)$ & $0,2(0,2 / 0,3)$ & 0.717 & - \\
\hline LOS & $49(37 / 58)$ & $53(40$ / 68) & $63(45 / 70)$ & $0.016 *$ & $0.008^{+}$for $1-3$ \\
\hline Eyes Open Firm Surface & $0,47(0,36 / 0,54)$ & $0,47(0,34 / 0,68)$ & $0,54(0,43 / 0,6)$ & 0.698 & - \\
\hline $\begin{array}{l}\text { Eyes Closed Firm } \\
\text { Surface }\end{array}$ & $0,55(0,48 / 0,75)$ & $0,55(0,48 / 0,75)$ & $0,79(0,51 / 1,31)$ & $0.032^{*}$ & $0.008^{+}$for $2-3$ \\
\hline $\begin{array}{l}\text { Eyes Open Foam } \\
\text { Surface }\end{array}$ & $0,99(0,79 / 1,16)$ & $0,91(0,64 / 1,05)$ & $0,87(0,71 / 1,16)$ & 0.234 & - \\
\hline $\begin{array}{l}\text { Eyes Closed Foam } \\
\text { Surface }\end{array}$ & $1,79(1,6 / 2,04)$ & $1,86(1,55 / 2,23)$ & $2,09(1,67 / 2,59)$ & 0.076 & - \\
\hline
\end{tabular}

APSI: Antero-Posterior Stability Index, MLSI: Medio-Lateral Stability Index, OSI: Overall Stabilite Index, LOS: Limit of Stability, ${ }^{*} p<0.05,{ }^{+}$New $p$ value with Bonferroni correction $\mathrm{p} / 3=0.05 / 3=0.016$ 


\section{DISCUSSION}

The aim of this longitudinal study was to evaluate pregnant women in terms of static balance and reveal differences during three trimesters. Contrary to expectations, it was seen that there was no significant change in postural stability. However, as expected, changes were detected in the last trimester in terms of sway tested in the LOS and in the mCTSIB.

When literature is examined, several studies evaluating postural stability during pregnancy were encountered. Ribas and Guirro stated that even though pregnant women in the third trimester showed greater AP displacement than those in the first trimester, there was no difference in $\mathrm{ML}$ displacement (12). In the mentioned study, different pregnant women were evaluated for each trimester and this may have led to different results from our study. Jang et al. evaluated pregnant women at 0, 4, 8, 12 weeks and at 6 months postpartum with the stabilogram diffusion analysis and compared the results with the control group. They found that postural sway in the AP direction increased during pregnancy and decreased after delivery. They also reported that sway in the ML direction varied little during pregnancy, but it tended to increase after delivery, and compared to the control group, these values were higher in pregnant women (2). In their review, Ribeiro et. al. put forth that postural stability decreased during pregnancy due to possible reasons such as adaptations developed in the spine and lower extremities. They stated that postural stability decreases gradually during pregnancy, this decrease continues for 6-8 weeks after delivery and the reduction in postural stability increases the risk of falls during pregnancy (13). Similar to our study, Mocellin and Driusso, who evaluated postural stability by assessing the same pregnant women over three trimesters, stated that postural control tended to decrease in the first trimester of pregnancy and decreased further in the third trimester, however this decrease was not statistically significant (14). In our study, there was no significant change in postural stability between the three trimesters. Postural control during standing involves the integration of sensory information from the whole body, in particular from mechanoreceptors on the soles of the feet and from specialized receptors which code body position and orientation with respect to the gravitational acceleration, the environment and the body segments. In other words, it is maintained as a result of the central control of visual, vestibular and proprioceptive inputs. Although postural control mechanisms appear to be unaffected during pregnancy, the increase and asymmetric distribution of body mass and posterior body tilt observed during pregnancy may play an important role in modulating the amplitude and frequency of body sway, reflecting certain strategies for maintaining upright standing posture (15). The body of a pregnant woman undergoes significant biomechanical changes in the sagittal plane, such as the posterior body tilt. The pregnant woman's posture adapts with a slight posterior body tilt to reduce muscular energy expenditure and maintain AP stability while standing $(5,7)$. The increase of mass in the anterior pelvic region in pregnant women is compensated for with the increase of tonic activity of ankle plantar flexors and the increase of ankle stiffness and the duration of body sways increases. Despite the larger amount of tonic ankle activity, women in the later stages of pregnancy would possibly compensate for body sways with modulations of ankle torque at lower frequencies (15). Furthermore, it is stated that pregnant women try to maximize their postural stability by adjusting their step width (14). These mechanisms may explain why postural stability may not have changed between trimesters in our study.

Our results show that the LOS vary between trimesters. LOS is defined as the maximum angle a person's body can achieve from vertical without losing balance. As a result of this study, it was observed that the LOS was increased in the third trimester when comparison to the first trimester. It should be remembered that a low 'actual' score obtained as a result of the LOS test indicates that the person's LOS have decreased. In their study performed to investigate the effects of a maternal support belt, Elena Bey et. al. compared the LOS of pregnant women in different trimesters during pregnancy with and without the belt. Researchers found that without the maternal support belt, the LOS in the first and third trimesters was significantly higher in both anterior and posterior directions than the control group. They stated that the LOS in the second trimester was slightly lower compared to other trimesters but did not differ from the controls. They found that the LOS performance decreased in the early stages of pregnancy before the body mass had increased. They explained that factors such as increased anxiety due to pregnancy could have affected postural stability rather than body mass (16). Our results may have differed from the study by Elena Bey et al, due to the fact that they evaluated different pregnant woman in each trimester. In our study, the reason for the increase in the LOS value may be due to the increase in the size of the abdomen of pregnant women, which enabled them to reach the target points in the test much more easily with less body movement. Furthermore, as it is known, CoG moves forward with the increase in the size of the abdomen. This may have enabled the pregnant women to have better control on the CoG and move it out of the base of support.

mCTSIB evaluates standing balance in different situations. It was seen that during the Eyes Closed Firm Surface test, which is one of the sub-parameters of MCTSIB, sway was increased in the third trimester compared to the second trimester. The main reason for this is the elimination of visual input. According to the sensory organization theory first reported by Nasher, the central nervous system encodes the information provided by visual, vestibular and somatosensory receptors (17). Although the effects of the absence of visual input on upright posture control in normal subjects are controversial, the results of this study propose that pregnant women tend to compensate for the lack of vision by increasing the frequency of sway. In the study carried out by Oliveira et al. healthy pregnant women were evaluated using a stabilometer during different visual situations (eyes open/closed) and in different support base 
configuration (feet together/apart). As a result, they found that postural control changed significantly during pregnancy when visual input was suppressed, or the support base was reduced. Therefore, they concluded that the accuracy of visual information and a comfortable support base could be critical for preserving balancing ability in the later stages of pregnancy (15). In their study, Butler et al. measured the eyes open and eyes closed static balance of pregnant women in 3 trimesters and compared the results with the control group. They stated that the second trimester, third trimester and post-pregnancy values were higher than the control group. Thus, they found that postural stability decreased during pregnancy and this decrease continued after delivery. This study also showed the increasing dependence on visual input to maintain balance during pregnancy (18). In their longitudinal study, Opala-Berdzik et al. evaluated pregnant women in their 1st trimester, 3rd trimester and postpartum. The authors concluded that static postural stability was not different between pregnancy and the postpartum period except AP sway, which was tested eyes-closed. This suggests that when visual input is eliminated, women in advanced pregnancy may have reduced static stability compared to non-pregnant women (5). These studies support our study as they show that sway is increased in eyes closed conditions.

Although static balance was evaluated in this study, some studies evaluating dynamic balance in pregnant women showed similar results with our study stating that stability indexes remained the same. McCrory et al. found that there was no difference in timing and magnitude of sway in response to perturbations in the second trimester, however, with progression from the second trimester to the third trimester, it was found that the sway responses decreased in pregnant women (19). Cakmak et al. found that without the support belt, there was no difference between trimesters in terms of OSI, MLSI and APSI, however the fall risk test scores were higher in the third trimester compared to other trimester (20). In their study, Inanir et al. reported that there was no significant difference in terms of OSI, APSI and MLSI between non-pregnant women and pregnant women in the first and second trimesters. They stated that pregnant women in the third trimester had higher OSI, APSI and MLSI scores than non-pregnant controls (21). The lack of difference in OSI, APSI and MLSI scores in these studies supports our study. However, the fact that Cakmak and Inanir evaluated different pregnant women in each trimester differentiates these studies from our study.

When literature is examined, there are different studies providing contradictory results regarding the change of balance in pregnant women. One reason for this may be that there are many different test methods used to assess balance in the clinic. Another reason is that when evaluating between trimesters, some studies included different pregnant women for each trimester, while in longitudinal studies the same pregnant woman were evaluated over three trimesters. The fact that many anatomical and physiological changes occur in pregnant women during pregnancy and these changes are seen in different rates in each pregnant woman may be another reason explaining the variety of results in the studies. Additionally, there is no standardization of evaluation time in these studies. Using different assessment methods at different time intervals may be among the reasons that can explain these conflicting results. Although the results of the studies vary, literature supports the fact that the balance of pregnant women will quite likely be affected due to many reasons mentioned above.

\section{CONCLUSION}

This study was carried out to examine the changes in static balance in pregnant women during pregnancy. It can be stated that this study contributes to the literature in terms of follow-up of the same pregnant woman for three trimesters and evaluation of static balance with different sub-parameters. It should be considered that balance performance during pregnancy may vary depending on the condition in which it was assessed and the degree of physical difficulty. This may explain why, in most studies, there was no difference between trimesters in a moderatelydifficult test performed in a static condition with eyes open. The assessment of postural balance during pregnancy can contribute to the development and application of therapeutic methods to prevent postural instability and falls. Although there is no consensus in literature regarding how balance changes in pregnant women, it is highly probable that the balance of pregnant women will be affected. Pregnant women should be especially educated against the risk of falling when in conditions such as darkness where vision is limited. Therapists should pay attention to changes in the static and dynamic postural control of pregnant women and create an exercise program that aims to improve balance by preventing falls, maintaining muscle strength, and physical well-being throughout pregnancy. In conclusion, we believe that multi-faceted evaluation, using different assessment methods and parameters may be beneficial in evaluating balance and risk of falls, and these factors should be taken into consideration when applying clinical postural tests to pregnant women.

\section{REFERENCES}

[1] Soma-Pillay P, Catherine N-P, Tolppanen H, Mebazaa A, Tolppanen $\mathrm{H}$, Mebazaa A. Physiological changes in pregnancy. Cardiovasc J Afr 2016;27(2):89.

[2] Jang J, Hsiao KT, Hsiao-Wecksler ET. Balance (perceived and actual) and preferred stance width during pregnancy. Clin Biomech 2008;23(4):468-476.

[3] Norwitz E, Robinson J, Repke J, Gabbe S, Niebyl J, Simpson J. Obstetrics; normal and problem pregnancies. 4th ed New York: Churchill Livingstone. 2002:353-394.

[4] Jensen R, Doucet S, Treitz T. Changes in segment mass and mass distribution during pregnancy. J Biomech 1996;29(2):251-256.

[5] Opala-Berdzik A, Błaszczyk JW, Bacik B, Cieślińska-Świder J, Świder D, Sobota G, Markiewicz A. Static postural stability in women during and after pregnancy: a prospective longitudinal study. PLoS One 2015;10(6): e0124207. 
[6] Bivia-Roig G, Lisón JF, Sanchez-Zuriaga D. Changes in trunk posture and muscle responses in standing during pregnancy and postpartum. PLoS One 2018;13(3): e0194853.

[7] Opala-Berdzik A, Bacik B, Cieślińska-Świder J, Plewa M, Gajewska M. The influence of pregnancy on the location of the center of gravity in standing position. J Hum Kinet 2010;26:511.

[8] Marnach ML, Ramin KD, Ramsey PS, Song S-W, Stensland JJ, An K-N. Characterization of the relationship between joint laxity and maternal hormones in pregnancy. Obstet Gynecol 2003;101(2):331-335.

[9] Cakmak B, Ribeiro AP, Inanir A. Postural balance and the risk of falling during pregnancy. J Matern Fetal Neonatal Med 2016;29(10):1623-1625.

[10] Ma J, Yao Y, Ma R, Li J-Q, Wang T, Li X, Han WQ, Hu WD, Ming Zhang Z. Effects of sleep deprivation on human postural control, subjective fatigue assessment and psychomotor performance. J Int Med Res 2009;37(5):1311-1320.

[11] Hinman MR. Factors affecting reliability of the Biodex Balance System: a summary of four studies. J Sport Rehabil 2000;9(3):240-252.

[12] Ribas S, Guirro E. Analysis of plantar pressure and postural balance during different phases of pregnancy. Rev Bras Fisioter 2007;11(5):391-396.

[13] Ribeiro AP, João SMA, Sacco ICN. Static and dynamic biomechanical adaptations of the lower limbs and gait pattern changes during pregnancy. Womens Health 2013;9(1):99-108.
[14] Moccellin A, Driusso P. Adjustments in static and dynamic postural control during pregnancy and their relationship with quality of life: A descriptive study. Fisioterapia 2012;34(5):196202.

[15] Oliveira LF, Vieira TM, Macedo AR, Simpson DM, Nadal J. Postural sway changes during pregnancy: a descriptive study using stabilometry. Eur J Obstet Gynecol Reprod Biol 2009;147(1):25-28.

[16] Bey ME, Arampatzis A, Legerlotz K. The effect of a maternity support belt on static stability and posture in pregnant and non-pregnant women. J Biomech 2018;75:123-128.

[17] Nashner L, editor Sensory, neuromuscular, and biomechanical contributions to human balance. Proceeding of APTA Forum, Tennessee 1989.

[18] Butler EE, Colón I, Druzin ML, Rose J. Postural equilibrium during pregnancy: decreased stability with an increased reliance on visual cues. Am J Obstet Gynecol 2006;195(4):1104-1108.

[19] McCrory J, Chambers A, Daftary A, Redfern M. Dynamic postural stability during advancing pregnancy. J Biomech 2010;43(12):2434-2439.

[20] Cakmak B, Inanir A, Nacar MC, Filiz B. The effect of maternity support belts on postural balance in pregnancy. PM $R$ 2014;6(7):624-628.

[21] Inanir A, Cakmak B, Hisim Y, Demirturk F. Evaluation of postural equilibrium and fall risk during pregnancy. Gait Posture 2014;39(4):1122-1125.

How to cite this article: Sancar S, Atalay Guzel N, Cobanoglu G, Ozdemir YA, Bayram M. The Changes in Static Balance During Pregnancy: A Prospective Longitudinal Study. Clin Exp Health Sci 2021; 11: 127-132. DOI: 10.33808/clinexphealthsci.744603 